\title{
A survey to establish current methods of venous thromboembolism prophylaxis in stroke patients practiced by Polish neurologists
}

Jan Paweł Bembenek ${ }^{1}$, Martin Dennis², Anna Czlonkowska ${ }^{1,3}$

\begin{abstract}
${ }^{1} 2^{\text {nd }}$ Department of Neurology, Institute of Psychiatry and Neurology, Warsaw, Poland ${ }^{2}$ Division of Clinical Neurosciences, Western General Hospital, Edinburgh, Scotland ${ }^{3}$ Department of Experimental and Clinical Pharmacology, Medical University of Warsaw, Warsaw, Poland
\end{abstract}

Submitted: 4 December 2012

Accepted: 27 March 2013

Arch Med Sci 2014; 10, 3: 470-476

DOI: 10.5114 /aoms.2014.43742

Copyright (c) 2014 Termedia \& Banach

\section{Abstract}

Introduction: Venous thromboembolism (VTE) is associated with considerable morbidity and mortality in stroke patients. The purpose of our survey was to establish the current methods of VTE prophylaxis practiced by Polish neurologists. We also aimed to determine whether there is enough variation in practice to justify the development of an evidence-based guideline for VTE prevention.

Material and methods: Postal self-administered questionnaires about VTE prophylactic methods practiced by Polish neurologists were sent to 218 neurological wards where stroke is treated. If no response was received, the questionnaire was faxed and finally we attempted to obtain information by telephone.

Results: One hundred and seventy-six (80.7\%) stroke centers of 218 responded. The majority $(137 / 176 ; 77.8 \%)$ of centers had a stroke unit. The median admission rate of surveyed wards was estimated to be 320 patients/ year (range from 20 to 1000 patients/year). The most common method of VTE prophylaxis reported was low molecular weight heparin (LMWH) (98.9\%), and the least common was intermittent pneumatic compression (IPC) (6.8\%). Centers admitting $\geq 200$ patients/year $(70.7 \%$ vs. $45 \% ; p=0.039)$, and those with stroke units ( 95 vs. $20 ; p=0.031$ ) were more willing to join a randomized trial evaluating any of the methods. All the centers participating in the survey reported a need for a detailed VTE prophylaxis guidelines.

Conclusions: The LMWH is the predominant VTE prophylaxis strategy for stroke patients practiced by Polish neurologists. Due to the variation of methods used in VTE prophylaxis it might be reasonable to further specify this section of stroke treatment guidelines.

Key words: stroke, deep venous thrombosis, venous thromboembolism, prophylaxis, pulmonary embolism, survey.

\section{Introduction}

Venous thromboembolism (VTE), including deep venous thrombosis (DVT) and its sequela, pulmonary embolism (PE), is an important cause of illness and death after stroke [1]. At greatest risk are patients who are immobile, with significant weakness of the limbs and a prior history of DVT [2]. Depending on the methodology of the studies, the frequency of

\section{Corresponding author:}

Jan Paweł Bembenek MD, PhD $2^{\text {nd }}$ Department of Neurology Institute of Psychiatry and Neurology

9 Sobieskiego St

02-957 Warsaw, Poland

Phone: +4822 4582872

Fax: +48228424023

E-mail: jbembenek@o2.pl 
DVT is up to $80 \%$ of patients with ischemic stroke who did not receive prophylactic therapy [3]. The frequency of above-knee DVT using Doppler ultrasound technique was lower ( 10\%) [4-6]. Additionally, the types of patients included and the duration and timing of follow-up influenced the estimates. Approximately $5 \%$ of early deaths following stroke are attributed to pulmonary embolism [1].

There are pharmacological (unfractionated heparin - UFH, low-molecular-weight heparins LMWH, oral anticoagulants, new oral anticoagulants, dextrans) and physical methods (graduated compression stockings (GCS), intermittent pneumatic compression devices (IPC)) which aim to reduce the risk of VTE $[7,8]$. The strength of evidence for each varies. There is no "gold standard" method for VTE prevention that is recommended in all post-stroke patients. Inevitably, where reliable evidence is not available, variation in recommendations exists [7, 9-11].

There are no reliable data regarding the variation in use of VTE prophylactic methods in Poland and the multiplicity of the available recommendations and guidelines can contribute to such diversity [7, 9-13]. However, it is important to find out whether such variation exists amongst Polish stroke units and neurological wards. In contrast to other countries such as England or Scotland, in Poland stroke is generally treated in stroke units, which are a part of neurological wards [13].

National surveys may bring interesting and valuable information about variation of clinical practice among the centers in the country, especially when there are discrepancies in data derived from the literature [14].

The aim of the study was to establish:

- whether Polish neurologists working in neurological wards and stroke units use VTE prophylaxis;

- what methods are used in each ward or unit;

- whether there is much variation in practice and whether it is sufficient to justify the development of an evidence-based guideline specifically for Polish neurologists;

- whether there is enough uncertainty regarding which method of VTE prophylaxis should be chosen in stroke that will encourage Polish neurologists to participate in randomized trials comparing different methods of prophylaxis practiced in Poland.

\section{Material and methods}

A questionnaire (see Supplement 1) was sent to all neurological wards listed in the Polish Ministry of Health as treating stroke. The questionnaire comprised five questions. The answers were yes or no, and the only response that required a definite number was an estimate of the number of stroke patients admitted per year. Heads of the departments/wards were asked: whether they have a stroke unit in their hospital; approximately how many stroke patients are admitted to their center per year; what method(s) they use in their departments (multiple choice option: UFH, $\mathrm{LMWH}$, oral anticoagulants, new oral anticoagulants, dextrans, hydration, early mobilization, GCS - full length or below knee, IPC); and also whether they use those methods routinely or in selected patients (when hemorrhagic stroke was excluded; only those at substantial risk of VTE development; individual decision for each patient). We also asked whether respondents would be interested in joining a randomized trial evaluating any of the prophylactic methods (interested in joining a trial evaluating any method: yes or not; and response options: UFH, LMWH, oral anticoagulants, dextrans, GCS, IPC). Additionally, respondents were asked if it would be useful to publish detailed Polish guidelines of VTE prevention in stroke patients (response option: yes or no).

The questionnaire was first sent by e-mail. If the centers did not respond to the survey, the questionnaire was sent by fax. The last attempt was to obtain data by telephone.

The survey was conducted between January and May 2012. Participation was voluntary and all responses were confidential. We received only one response from each center (usually provided by a Head of the Department).

As well as reporting the overall results we also compared the responses of the centers with a stroke unit with those without one and centers admitting $\geq 200$ patients/per year with those admitting < 200 patients/per year.

\section{Statistical analysis}

The mean, range and percentage were noted for descriptive summary statistics. Nominal variables were compared using the $\chi^{2}$ test or two-sided exact Fisher's test if the expected value was $\leq 5$. A $p$ value $<0.05$ was considered statistically significant. Calculations were carried out in Statistica 10.0 PL (StatSoft, Inc. 2011).

\section{Results}

Of 218 neurological wards and/or stroke units in Poland, 176 (80.7\%) responded to our survey. The majority of the centers participating in the survey had stroke units (137/176, 77.8\%). The median number of estimated stroke hospitalizations was 320 patients/year (range from 20 to 1000 patients/year).

All centers reported the use of several methods of VTE prophylaxis (Table I). Low molecular weight 


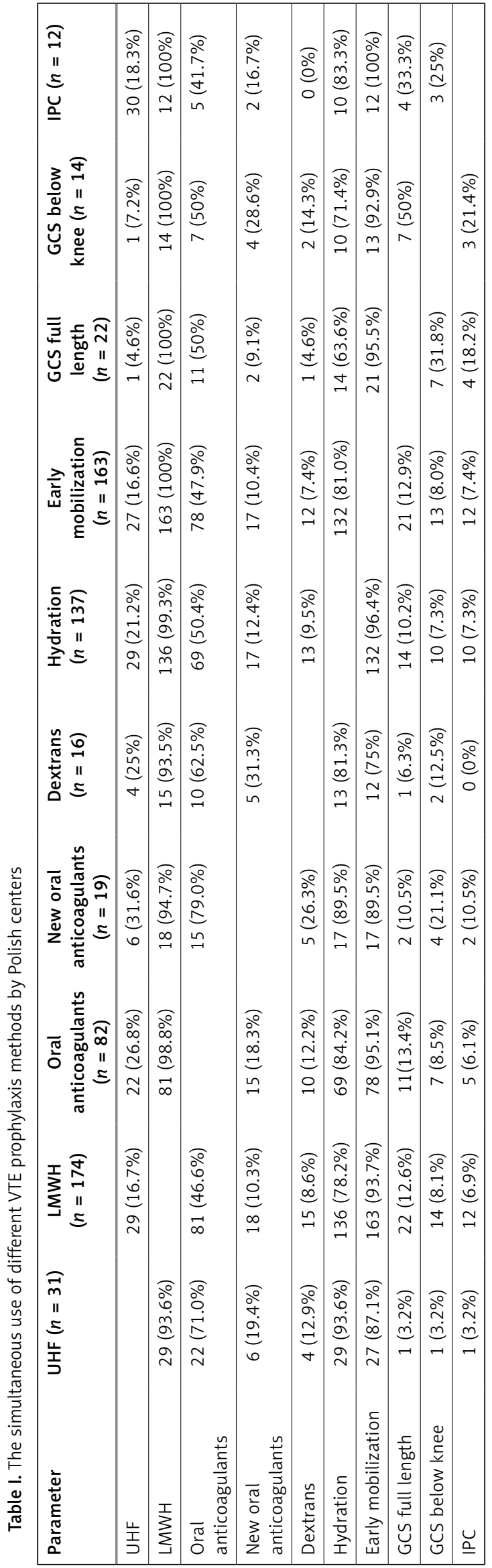

heparin was the predominant VTE prophylactic strategy (174/176, 98.9\%). The least frequently used were IPC devices (12/176, 6.8\%). A detailed presentation of frequency of the methods used is provided in Figure 1.

The majority of centers used prophylactic methods routinely in all immobile stroke patients (105/176, 59.7\%). Others used prophylaxis (this presumably mainly referred to anticoagulants) routinely only in those in whom intracranial hemorrhage had been excluded $(62 / 176,35 \%)$ or only in those thought to be at particularly high risk of VTE development (13/176, 7.3\%). In 8/176 (4.5\%) centers the decision on the choice of prophylactic method was based on other factors.

The majority of centers (115/176, 65\%) were interested in joining a randomized trial evaluating any of the prevention methods of VTE in stroke patients. Six centers did not answer this question. Large centers were more interested in taking part in such a study than smaller ones $(70.7 \%$ vs. $45 \%$, $p=0.039$ ). Most of the surveyed centers were interested in joining a randomized trial evaluating LMWH (111/170, 63\%). The centers willing to participate in the randomized trial were less interested (24/170, 13.6\%) in joining a trial evaluating the safety and efficacy of dextrans. A detailed presentation of the preferred methods of prevention among centers that would be interested in evaluating such methods in a randomized study $(n=$ $115)$ is provided in Figure 2.

We performed a comparative analysis of centers with a stroke unit (132/176) and those without $(38 / 176)$. The only method practiced by centers with a stroke unit that was statistically more frequently used was early mobilization (94.9\% vs. $84.6 \%, p=0.04)$. Dextrans were less frequently used in centers with a stroke unit $(6.6 \%$ vs. $13 \%$, $p=0.05)$. The centers with a stroke unit were also more willing to join a randomized trial evaluating any of the methods used in VTE prophylaxis in stroke patients (95/132 vs. 20/38; $p=0.031)$, but there were no statistical differences regarding the methods of prevention that centers would like to assess.

Additionally we compared the centers admitting < 200 patients/year with those admitting $\geq 200$ patients/year. New oral anticoagulants were more frequently (214/156 vs. 5/20) used in centers admitting less than 200 patients per year, which was statistically significant $(p=0.046)$. There were no other statistical differences between centers admitting $\geq 200$ and $<200$ patients per year in terms of using other methods of prophylaxis or being willing to participate in a trial evaluating those methods $(p>0.05)$. Centers admitting $\geq 200$ per year were more interested in joining randomized trials evaluating any of the 


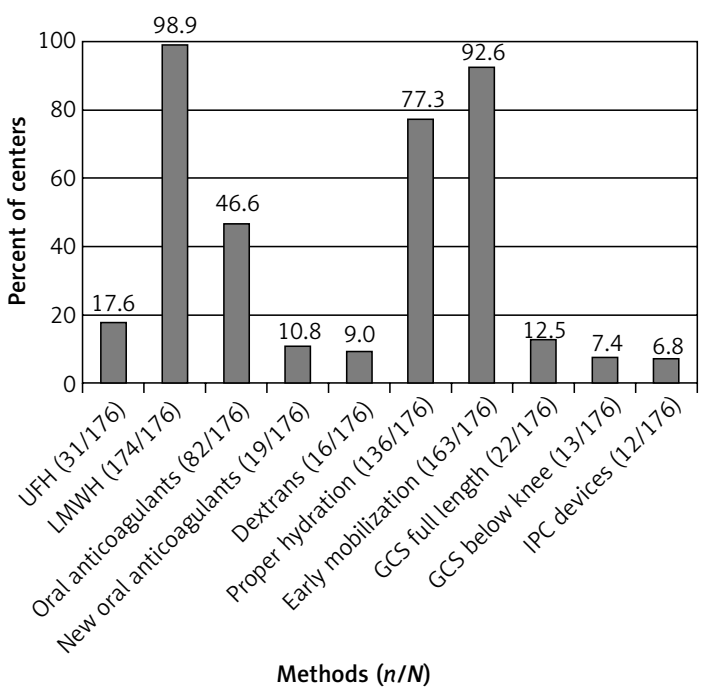

LMWH - low molecular heparins, GCS - graduated compression stockings, IPC - intermittent compression devices, UFH - unfractioned heparine

Figure 1. Methods used for VTE prevention in stroke patients in Polish centers that responded to the survey

methods used in VTE prophylaxis (70.67 vs. 45; $p=0.039$ ), but no statistical differences in terms of particular methods were found.

\section{Discussion}

In this survey representing practice patterns in 176 Polish neurological wards and/or stroke units we found that all centers used several methods of VTE prophylaxis in stroke patients. Low molecular weight heparin was the dominant method for prophylaxis, although there are no randomized trials comparing the use of $\mathrm{LMWH}$ vs. placebo to prevent VTE in stroke patients. Both American and European guidelines suggest the use of heparins (UFH and $\mathrm{LMWH}$ ) in VTE prevention in stroke patients, but suggest their use mostly in those considered to be at high risk of VTE and low risk of bleeding - interpretation of this recommendation may be problematic and controversial [7, 9-11, 13]. The grade of the recommendation varies, which may result from the different interpretation of the evidence and the differences in historical choices in those countries.

Early mobilization was practiced in $92.6 \%$ of centers. Although there is an ongoing debate about the definition and effectiveness of this method [15], it is recommended by European Stroke Organization (ESO) guidelines [12], as well as by the newest Polish guidelines of stroke treatment and prevention published in 2012 [13]. The latest American guidelines do not mention the use of this method [7]. There are no trials that have evaluated the effectiveness of this method in VTE

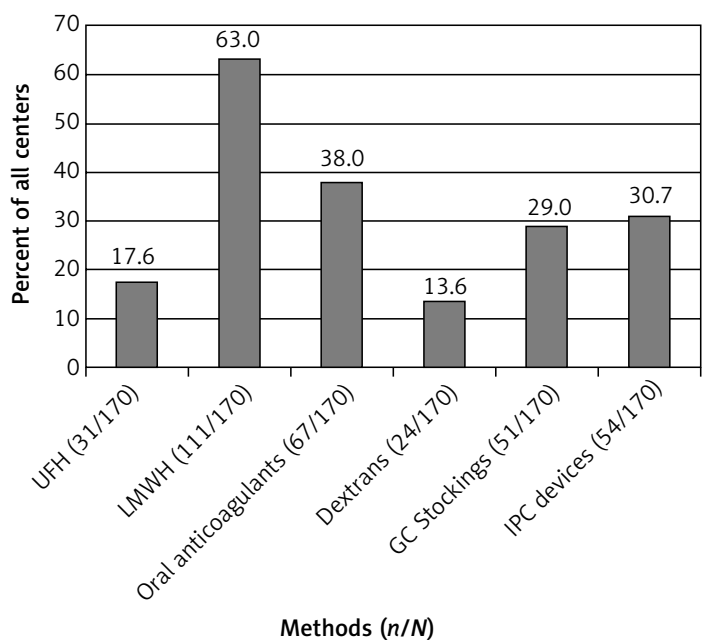

LMWH - low molecular heparins, GCS - graduated compression stockings, IPC - intermittent compression devices, GC - graduated compression

Figure 2. Preferred methods of VTE prevention to be evaluated among centers willing to participate in the randomized study evaluating such methods $(n / N=115 / 170,65 \%)$.

prevention in stroke patients. Moreover, the ongoing AVERT trial (A Very Early Rehabilitation Trial for Stroke) will also not answer whether this method is effective in VTE prevention as it is trying to test its effect on functional outcomes but not VTE [16].

In the majority of the Polish centers $(72.7 \%$ of centers) good hydration is a part of VTE prevention. Although the effectiveness of this method in stroke patients is based only on one small observational study [17], both European and Polish guidelines recommend good hydration of stroke patients [12, 13]. Kelly et al. established that a marker of dehydration was associated with more frequent DVT, but the study did not show that improving hydration reduces the incidence of VTE [17]. The Cochrane systematic review of hemodilution did show a possible effect on DVT rate but this was indirect evidence [18].

The GCS were reported to be less frequently used by our respondents. Two large clinical trials aimed to determine whether GCS effectively reduce the risk of DVT in stroke patients. CLOTS 1 (Clots in Legs Or sTockings after Stroke) [5] was designed to evaluate the effectiveness of thighlength GCS $(n=1256)$ vs. routine care $(n=1262)$. Proximal DVT was evaluated on compression duplex ultrasound examination at 7-10 days and 2530 days after stroke. It was the primary end point and was found in $10.0 \%$ and $10.5 \%$ of patients, respectively, which resulted in a non-significant absolute reduction in risk of $0.5 \%(95 \% \mathrm{Cl}-1.9 \%$ to $2.9 \%$ ). Moreover, stockings significantly increased the risk of skin breaks, ulcers, blisters, and skin necrosis (64 (5\%) vs. 16 (1\%); odds ratio 4.18, 
95\% Cl: 2.40-7.27). The CLOTS trial 2 compared the effectiveness of thigh-length stockings with that of below-knee stockings for preventing proximal DVT in 3114 immobile, hospitalized patients with stroke. The DVT occurred more often in patients who received thigh-length stockings ( $p=0.008$ ) [19]. Despite the results of CLOTS 1 and CLOTS 2 trials, this method of prophylaxis is still used in approximately $20 \%$ of Polish centers (both full length and below knee stockings). The newest version of the Polish guidelines do not recommend the routine use of GCS in post-stroke patients (Grade 2) [13]. National Institute of Health and Clinical Excellence (NICE), Scottish Intercollegiate Guidelines Network (SIGN), American College of Physicians (ACP) and American College of Chest Physicians (ACCP) guidelines recommend that GCS should not be used in stroke patients [7, 9-11].

Although GCS have been proven not to be effective in DVT prevention in stroke patients [5], $30.7 \%$ of all the surveyed centers declared willingness to participate in the study if it was carried out. There were no statistical differences between large and small or stroke unit and no stroke unit centers. It is worth noting that before the publication of CLOTS 1 trial results, the use of compression stockings was a widely recommended method of VTE prophylaxis. There is also a "tradition" or a habit of their use based on their effectiveness in surgical patients [20], which was probably extrapolated to patients with stroke. Nevertheless, in the United Kingdom publication of the results of the CLOTS trials had a dramatic impact on clinical practice [21].

Although there is insufficient evidence to support the routine use of pneumatic compression devices to prevent VTE in stroke patients [8], this method is still practiced in some Polish neurological wards and/or stroke units (6.8\%). This method has never been commonly practiced by Polish neurologists. The results of the large randomized trial CLOTS 3, which compares the effectiveness of IPC vs. None in 2876 stroke patients, will be available in May 2013 [22]. The IPC is recommended by both European and American guidelines (Grade $2 \mathrm{c}$ recommendation) $[7,12]$. Polish guidelines do not mention this method of VTE prophylaxis [13].

As all the centers that claimed to mobilize early and use GCS or IPC were also using LMWH, and only one center claiming to use hydration did not use LMWH, this may suggest that centers that are most interested in VTE prophylaxis use a variety of physical and pharmacological methods.

All the respondents (100\%) considered that it would be useful to publish guidelines for VTE prevention in post-stroke patients in Polish (this would facilitate access for people who do not know the English language). The existing guide- lines [13] do not address all the questions posed by Polish neurologists: they do not precisely describe the criteria of VTE prevention implementation, the time of onset and the duration of the use of those methods. The ESO guidelines for VTE prophylaxis, which are currently being developed, may answer these questions.

The fact that centers with a stroke unit less frequently used dextrans in VTE prophylaxis (no data for the effectiveness of this method), and the more frequent early mobilization may suggest that those centers are more familiar with current literature and guidelines of stroke care.

Due to the variation of methods used in VTE prophylaxis, it might be reasonable to publish detailed national guidelines for VTE prophylaxis in stroke patients. Such guidelines may help standardize the prophylaxis in stroke patients. They should of course be based on the results of systematic reviews and large clinical trials. We used evidence from two randomized trials to conduct this survey, suggesting that closed-ended formats yield more complete and valid demographic data than open-ended formats [23], and that appending second questionnaires to reminders maximizes response rates [24]. Our response rate was acceptably high and our findings are generalizable to neurological/stroke centers across Poland.

The response rate was acceptable (80.7\%), although not all centers responded to the questionnaire. We did not elicit information on the practice of combination prophylaxis with pharmacologic and nonpharmacologic approaches.

Respondents (mainly heads of departments) may not be aware of all local decisions, although their responses are probably representative of care standards delivered in their centers. The respondents may not be representative of all neurologists and their clinical practices. This limitation underscores the universal caveat of all surveys stating that practice does not always reflect actual practice.

Despite the limitations, the results of this survey represent the frequency of VTE prevention methods practiced by Polish neurologists and entirely justify the development of evidence-based guidelines or translation into Polish and adaptation of European ones.

In conclusion, our survey identified $\mathrm{LMWH}$ as the most common method of VTE prophylaxis for stroke patients practiced by Polish neurologists. The variation of methods used in VTE prophylaxis justifies the need for detailed guidelines for VTE prophylaxis in stroke patients.

\section{References}

1. Geerts WH, Bergqvist D, Pineo GF, et al. American College of Chest Physicians. Prevention of venous throm- 
boembolism: American College of Chest Physicians Evidence-Based Clinical Practice Guidelines (8th Edition). Chest 2008; 133: 381S-453S

2. Dennis M, Sandercock P, Reid J, et al. CLOTS Trials Collaboration. Can clinical features distinguish between immobile patients with stroke at high and low risk of deep vein thrombosis? Statistical modelling based on the CLOTS trials cohorts. J Neurol Neurosurg Psychiatry 2011; 82: 1067-73.

3. André C, de Freitas GR, Fukujima MM. Prevention of deep venous thrombosis and pulmonary embolism following stroke: a systematic review of published articles. Eur J Neurol 2007; 14: 21-32.

4. Oczkowski WJ, Ginsberg JS, Shin A, Panju A. Venous thromboembolism in patients undergoing rehabilitation for stroke. Arch Phys Med Rehabil 1992; 73: 712-6.

5. The CLOTS Trials Collaboration. Effectiveness of thighlength graduated compression stockings to reduce the risk of deep vein thrombosis after stroke (CLOTS trial 1): a multicentre, randomised controlled trial. Lancet 2009; 373: $1958-65$

6. Bembenek JP, Czlonkowska A, Karlinski M, Kobayashi A. Early stroke-related DVT is more than just DVT diagnosed early after stroke onset. Thromb Res 2011; 128: 587-9.

7. Lansberg MG, O'Donnell MJ, Khatri P, et al. American College of Chest Physicians. Antithrombotic and thrombolytic therapy for ischemic stroke: Antithrombotic Therapy and Prevention of Thrombosis, 9th ed: American College of Chest Physicians Evidence-Based Clinical Practice Guidelines. Chest 2012; 141 (Suppl 2): e601S-36S.

8. Naccarato M, Chiodo Grandi F, Dennis M, Sandercock PA. Physical methods for preventing deep vein thrombosis in stroke. Cochrane Database Syst Rev 2010; 8 CD001922.

9. National Institute for Health and Clinical Excellence. Venous thromboembolism: reducing the risk - full guideline. 2012; Available at: http://guidance.nice.org.uk/CG92.

10. SIGN Guideline 118. Management of patients with stroke: rehabilitation, prevention and management of complications, and discharge planning. Available at: http://www.sign.ac.uk/pdf/sign118.pdf

11. Qaseem A, Chou R, Humphrey LL, et al. Venous thromboembolism prophylaxis in hospitalized patients: a clinical practice guideline from the American College of Physicians. Ann Intern Med 2011; 155: 625-32.

12. The European Stroke Organisation (ESO) Executive Committee and the ESO Writing Committee. Guidelines for Management of Ischaemic Stroke and Transient Ischaemic Attack 2008. Cerebrovasc Dis 2008; 25: 457-507.

13. Postępowanie w udarze mózgu. Wytyczne Grupy Ekspertów Grupy Ekspertów Sekcji Chorób Naczyniowych Polskiego Towarzystwa Neurologicznego [Polish]. Neurol Neurochir Pol 2012; 46 (Suppl.1): S1-122.

14. Coutinho JM, Seelig R, Bousser MG, et al. Treatment variations in cerebral venous thrombosis: an international survey. Cerebrovasc Dis 2011; 32: 298-300.

15. Sundseth A, Thommessen B, Rønning OM. Outcome after mobilization within 24 hours of acute stroke: a randomized controlled trial. Stroke 2012; 43: 2389-94.

16. Sorbello D, Dewey HM, Churilov L, et al. Very early mobilisation and complications in the first 3 months after stroke: further results from phase II of A Very Early Rehabilitation Trial (AVERT). Cerebrovasc Dis 2009; 28: 378-83.

17. Kelly J, Hunt BJ, Lewis RR, et al. Dehydration and venous thromboembolism after acute stroke. QJM 2004; 97 293-6.
18. Asplund K. Haemodilution for acute ischaemic stroke. Cochrane Database Syst Rev 2002; 4: CD000103.

19. CLOTS (Clots in Legs Or sTockings after Stroke) Trial Collaboration. Thigh-length versus below-knee stockings for deep venous thrombosis prophylaxis after stroke: a randomized trial. Ann Intern Med 2010; 153: 553-62.

20. Wells PS, Lensing AWA, Hirsh J. Graduated compression stockings in the prevention of postoperative venous thromboembolism: a meta analysis. Arch Intern Med 1994; 154: 67-72.

21. Sett A, Mistri A. A dramatic impact of the CLOTS Studies on clinical practice in the UK. Int J Stroke 2011; 6: 15.

22. Dennis M, Sandercock P, Reid J, et al. CLOTS Trials Collaboration. Does intermittent pneumatic compression reduce the risk of post stroke deep vein thrombosis? The CLOTS 3 trial: study protocol for a randomized controlled trial. Trials 2012; 13: 26.

23. Griffith LE, Cook DJ, Guyatt GH, Charles C. Comparison of open versus closed questionnaire formats in obtaining demographic information from Canadian general internists. J Clin Epidemiol 1999; 52: 997-1005.

24. Asch DA, Jedrziewski MK, Christakis NA. Response rates to mail surveys published in medical journals. J Clin Epidemiol 1997; 50: 1129-36. 
Supplement 1.

\section{Questionnaire}

Filling in this questionnaire will take you about 2 minutes. After filling in the questionnaire please send it back to the e-mail address jbembenek@yahoo.com or put it in the included stamped addressed envelope and send it to the following address:

$2^{\text {nd }}$ Department of Neurology

Institute of Psychiatry and Neurology

Ul. Sobieskiego 9

02-957 Warsaw

Please write your name, name of hospital, and voivodship. If you put your e-mail address we will send you the results of the survey:

1) Do you have a stroke unit in your hospital?

Yes No

2) Which of the following methods do you use, either routinely or in selected patients, in stroke patients? You may put more than one tick.

a) UFH - unfractionated heparin

b) LMWH - low-molecular-weight heparin

c) Oral anticoagulants

d) New oral anticoagulants

e) Dextrans

f) Hydration

g) Early mobilization

h) Graduated compression stockings

1) Full length

2) Below knee

i) Intermittent pneumatic compression devices

Please indicate whether you use each of these methods.

- Routinely, in all immobile stroke patients

- Routinely in those in whom hemorrhage has been excluded

- Only those thought to be at particularly high risk

- Other option - please specify

3) Would you be interested in joining a randomized trial evaluating the efficacy of the following?

a) UFH - unfractionated heparin

b) LMWH - low-molecular-weight heparin

c) Oral anticoagulants

d) Dextrans

e) Graduated compression stockings

f) Intermittent pneumatic compression devices

4) How many stroke patients do you admit a year? Put the approximate number, please.

5) Do you think it would be useful to develop some Polish guidelines for preventing DVT after stroke? Yes No

Thank you very much for your co-operation. 